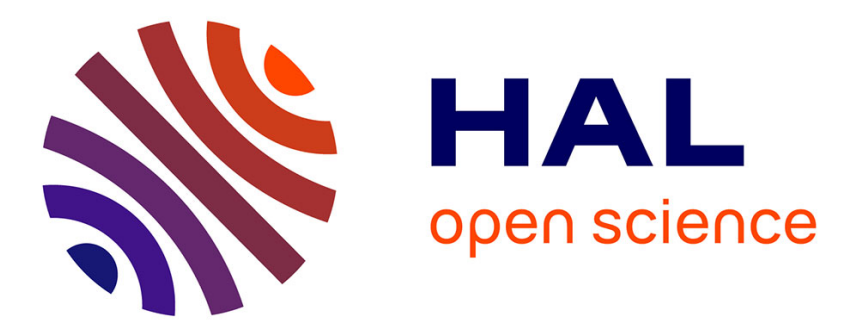

\title{
Wall slip regimes in jammed suspensions of soft microgels
}

Justin Péméja, Baudouin Géraud, Catherine Barentin, Marie Le Merrer

\section{To cite this version:}

Justin Péméja, Baudouin Géraud, Catherine Barentin, Marie Le Merrer. Wall slip regimes in jammed suspensions of soft microgels. Physical Review Fluids, 2019, 4 (3), pp.033301. 10.1103/PhysRevFluids.4.033301 . hal-02087121

\section{HAL Id: hal-02087121 \\ https://hal.science/hal-02087121}

Submitted on 1 Apr 2019

HAL is a multi-disciplinary open access archive for the deposit and dissemination of scientific research documents, whether they are published or not. The documents may come from teaching and research institutions in France or abroad, or from public or private research centers.
L'archive ouverte pluridisciplinaire HAL, est destinée au dépôt et à la diffusion de documents scientifiques de niveau recherche, publiés ou non, émanant des établissements d'enseignement et de recherche français ou étrangers, des laboratoires publics ou privés. 


\title{
Wall slip regimes in jammed suspensions of soft microgels
}

\author{
Justin Péméja, ${ }^{1}$ Baudouin Géraud,${ }^{1}$ Catherine Barentin,,${ }^{1,2}$ and Marie Le Merrer ${ }^{1, *}$ \\ ${ }^{1}$ Université de Lyon, Université Claude Bernard Lyon 1, \\ CNRS, Institut Lumière Matière, F-69622, Villeurbanne, France \\ ${ }^{2}$ Institut Universitaire de France, France
}

(Dated:)

\begin{abstract}
We characterize microfluidic flows of jammed suspensions of soft microgels (Carbopol) behaving as yield-stress fluids. We quantify the wall slip friction, i.e. the slip velocity $V$ versus the tangential stress at the wall $\sigma_{w}$. We demonstrate a transition in slip regimes, from a non-linear behavior $\left(V \propto \sigma_{w}^{2}\right)$ to a linear one, as the stress at the wall is increased, as expected from scaling arguments. Using fluorescent imaging to characterize the microgel size, we rationalize the two friction regimes for various samples by estimating viscous and elastic forces at the scale of the microgel particle. Only local arguments are thus necessary to predict wall slip friction, in contrast to other complex flow features such as fluidity or shear banding where bulk and surface properties appear to be strongly related.
\end{abstract}

\section{INTRODUCTION}

Foams, emulsions or microgel suspensions are soft glasses, constituted of a jammed assembly of soft objects in a liquid matrix — bubbles, droplets or polymer blobs. Macroscopically, they all behave as yield stress fluids: they are elastic-like at low stresses $\sigma$, but they flow at stresses larger than the yield stress $\sigma_{Y}[1-$ $3]$. Beyond their complex bulk rheology [3], the flow of these materials is known to be affected by the presence of solid walls, through local fluidization for instance $[4,5]$. In particular, it is widely observed that the classical no-slip boundary condition at the solid-liquid interface (continuity of tangential velocities) is broken, a phenomenon referred to as wall slip $[6,7]$. It is quantified by the relation between the velocity discontinuity at the wall (slip velocity $V$ ) and the stress tangential to the boundary $\sigma_{w}$. Microscopically, this phenomenon originates from the presence of a layer of interstitial liquid between the soft objects and the wall, which is preferentially sheared [6]. Strategies to avoid wall slip then consists in trapping the soft objects at the wall, either with a physical roughness comparable to the size of soft particles [8] or by suppressing the interstitial solvent layer with a strong attraction between the soft objects and the wall [7]. Otherwise, wall slip is ubiquitous in flows of yield-stress fluids.

In steady state, the slip velocity $V$ is found to increase with the wall stress $\sigma_{w}$. In dilute emulsions or microgel suspensions, the velocity increases linearly with the stress, as can be expected from a classical Stokes viscous friction $[9,10]$. The picture is more complicated in

\footnotetext{
* Corresponding author: marie.le-merrer@univlyon1.fr
}

concentrated materials exhibiting a yield stress, above the jamming point. First, some experiments have evidenced a wall yield stress [7, 10], below which no slip occurs. Whether this apparent wall yield stress is intrinsic [11] or an experimental artefact remains an open question [12], which we do not adress in this study. Above the apparent wall yield stress $\sigma_{w}^{Y}$, the slip velocity generally varies as a power law $V \propto \sigma_{w}^{p}$ or $\left(\sigma_{w}-\sigma_{w}^{Y}\right)^{p}$ where the exponent $p$ varies from 1 to 2 , depending on the complex fluids and the experimental conditions.

In foams, $p$ is found to be $\approx 2,3 / 2$ or 1 , depending on the liquid fraction and the physicochemistry of the foaming solutions [13-16]. The non-linear behaviors then arise from the coupling between surface tension and viscous effects in the liquid. In emulsions and microgel suspensions, either a linear $[8,12,17]$ or a square dependency of $V$ upon $\sigma_{w}[5,9,10,18-$ 20 ] is evidenced, depending on the stress and the chemical nature of the solid substrate, with respect to the one of the soft spheres. In addition, it has been suggested that the friction exponent switches from $p=2$ to 1 as the wall stress $\sigma_{w}$ becomes higher than the bulk yield stress $\sigma_{Y}$ [17], but other measurements have evidenced a non-linear scaling above the yield stress $[5,10,21]$. In this context, the aim of this article is first to quantify the influence of both the wall stress and the velocity on the value of the exponent $p$ by exploring many decades, and second to rationalize the friction regimes in terms of local dissipation mechanisms (figure 1). 


\section{MODELS OF WALL FRICTION OF SOFT SPHERE SUSPENSIONS}

The non-linear square behavior has been first explained by Meeker et al. [18] as follows. The soft particles are squeezed against the solid wall by the osmotic or confinement pressure $\Pi$ of the suspension. This pressure quantifies the 'degree of jamming' of the suspension; in particular, it vanishes when the polymer concentration $c$ is decreased down to the jamming point $c^{*}[22-$ 27 . This squeezing results in the formation of a thin liquid film of extension $r$ given by Hertz law: $r \sim R\left(\Pi / G_{P}\right)^{1 / 3}$ where $R$ and $G_{P}$ are the radius and the elastic modulus of the soft particle (Fig. 1). In this model, viscous dissipation is assumed to be dominated by shear flow in the thin film (blue region in Fig. 1). The average wall stress $\sigma_{w}$ is finally related to the viscous shear stress in the film $\eta V / \delta$, where $\eta$ is the dynamic viscosity of the interstitial liquid, and scales as: $\sigma_{w} \sim(r / R)^{2} \eta V / \delta$.

A crucial parameter here is the thickness $\delta$ of the thin liquid film: at very low velocity, it is fixed by the balance between elastic and surface (DLVO) forces [28, 29], resulting in a constant thickness $\delta$ and a linear friction regime, as recently observed on smooth silicon-silica surfaces [12]. However, at large enough velocities, viscous forces overcome surface ones. The thickness $\delta$ then depends on the slip velocity and derives from the balance between hydrodynamic and elastic forces [18]: $\delta^{2} \sim \eta R V / G_{P}$, at the origin of the non-linear friction regime. Indeed the wall stress reads: $\sigma_{w} \sim \sigma_{\mathrm{EHD}} \sim(\eta V / R)^{1 / 2} G_{P}^{1 / 2}\left(\Pi / G_{P}\right)^{2 / 3}$.

As the microgel concentration is decreased on the way to unjamming, corresponding to a left shift in the diagram of Fig. 1 (from case (1) to case (2)), the ratio $\Pi / G_{P}$ vanishes, so that both the extension of the thin films $r$ and the elastohydrodynamic friction stress $\sigma_{\mathrm{EHD}}$ tend to zero. For unjammed systems, one then expects that dissipation at the wall is determined by the shear in the liquid junctions of the microgel packing (red color on figure 1). The typical length scale for liquid shear is then given by the particle radius $R$ only, which yields a wall stress analogue to a Stokes law $\sigma_{w} \sim \sigma_{\mathrm{St}} \sim \eta V / R$. This change of scaling law was indeed observed by Divoux et al. [10] for thermo-responsive microgels across the jamming transition, and similar transitions in flow regimes were also observed in wet (close to unjamming) foams [16].

Comparing both elastohydrodynamic and Stokes terms shows that the linear regime

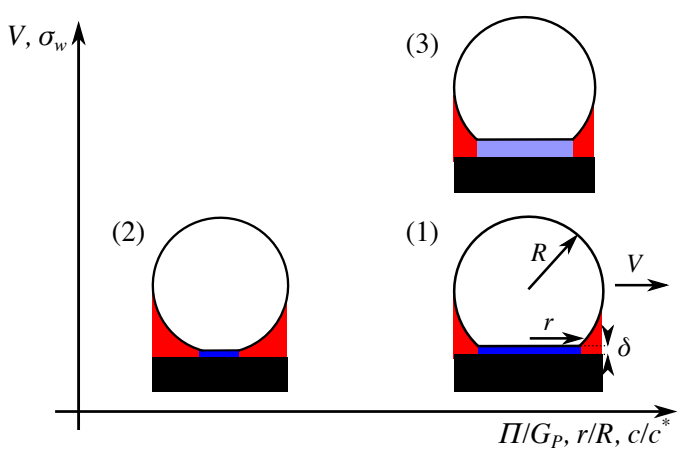

Figure 1. Sketch of the different configurations depending on the jamming degree ( $x$-axis) and on the wall stress or velocity ( $y$-axis). Blue: dissipation in the film (elastohydrodynamic friction), red: dissipation in the liquid junction (Stokes friction).

should dominate when $\sigma_{\mathrm{St}} \gg \sigma_{\mathrm{EHD}}$, that is for

$$
\sigma_{w} \gg G_{P}\left(\frac{\Pi}{G_{P}}\right)^{4 / 3} \text { or } V \gg \frac{R G_{P}}{\eta}\left(\frac{\Pi}{G_{P}}\right)^{4 / 3}
$$

Unjamming a microgel suspension means decreasing the normalized confinement pressure $\Pi / G_{P}$, hence lowering the right-hand side of Eq.1, which favors the Stokes-like regime (at a given wall stress or velocity). But for a given jammed fluid, i.e. a given pressure $\Pi$, Eq.1 also predicts that the friction regime should also change with increasing slip velocity $V$ : As $\delta$ increases with $V$, dissipation in the film (blue zone in figure 1) increases slower than the one in the liquid junctions (red zone). Eq.1 thus predicts a transition from an elastohydrodynamic regime to a Stokes regime with increasing velocity.

\section{WALL SLIP MEASUREMENTS}

To investigate the existence of a regime transition at higher velocity (from case (1) to case (3) of figure 1) and quantify the linear regime, we thus performed wall slip measurements of Carbopol suspensions to investigate over several decades the influence of the velocity on the value of the exponent $p$. Carbopol (Lubrizol) microgel suspensions consist in crosslinked polyacrylic acid blobs dispersed in water or water-glycerol mixtures. Once neutralized with sodium hydroxide, the polymer chains are negatively charged and repel each other, so that the polymer blobs swell and jam, which results into macroscopic yield stress $\sigma_{Y}$ and elastic modulus $G$. In this study, we use different Carbopol types (ETD 2050, Ultrez 10 (U10) 


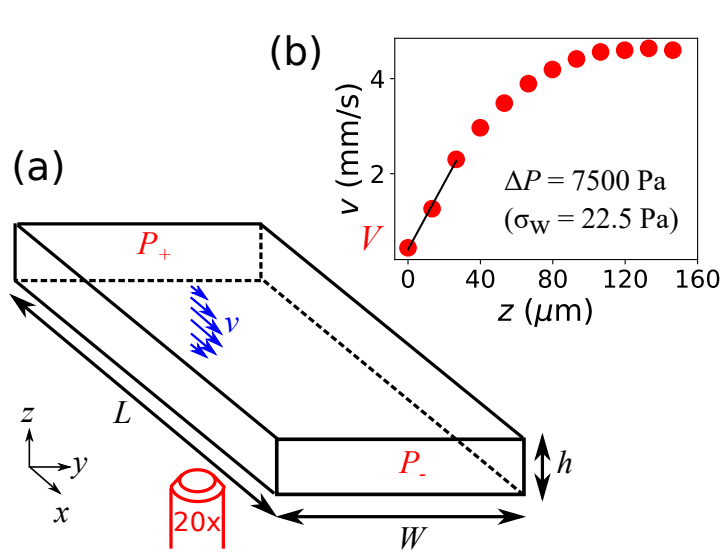

Figure 2. (a) Sketch of the experimental setup. The fluid velocities are measured from below with a microPIV setup [5]. (b) Typical flow profile $v(z)$ across one half of the capillary (Carbopol ETD $20500.25 \%, \sigma_{Y}=$ $1.6 \mathrm{~Pa}$ ). The systematic error on $v$ is $\sim 0.02 \mathrm{~mm} / \mathrm{s}$, much smaller than the symbol size.

and 980), corresponding to small changes in the formulation and cross-linking degree, following a preparation protocol described elsewhere $[5,30]$. The Carbopol weight concentration $c$ ranges from 0.1 to $1 \mathrm{wt} \%$. Note that, unless specified, all samples have been strongly mixed during 24 hours with a mixer (IKA, RW20). All samples behave as yield-stress fluids, whose rheology is well described by Herschel-Bulkley law which relates the shear stress $\sigma$ to the shear rate $\dot{\gamma}: \sigma=\sigma_{Y}+K \dot{\gamma}^{n}$, where $\sigma_{Y}$ is the yield stress, $K$ the consistency and $n$ the exponent. All rheological parameters are summarized in table I.

To measure wall slip, we take advantage of the transparency of the microgel suspensions which are seeded by $1 \mu \mathrm{m}$ fluorescent particles (Invitrogen) at volumic concentration $10^{-5}$ and characterize their flow in smooth glass capillary channels through a micro-Particle Image Velocimetry ( $\mu$ PIV) setup similar to the one used in [5]. The inner roughness of the microchannel has been measured by AFM and is characterized by a rms value of $0.2 \mathrm{~nm}$, much smaller than other lengthscale relevant here. The flow geometry is sketched in figure $2 \mathrm{a}:$ a pressure difference $\Delta P$ in the range $5 \cdot 10^{2}-1.8 \cdot 10^{5} \mathrm{~Pa}$ is applied by a pressure controller (Elveflow) and drives the Carbopol suspension through a rectangular glass capillary (VitroCom) of length $L=50 \mathrm{~mm}$, width $W=3 \mathrm{~mm}$ and height $h=0.3 \mathrm{~mm}$. The $W / h=10$ aspect ratio ensures that the flow can be considered as 2D along the $y$ direction. The stress across the channel is then known as: $\sigma(z)=\sigma_{x z}(z)=\Delta P(h / 2-z) / L$ (taking the origin $z=0$ at the lower wall defined in figure 2a). Finally, for different $z$ positions, images of the fluorescent particles are recorded. We use image correlation to detect their displacement from which the fluid velocity $v(z)$ is deduced. With our LaVision Imager Pro camera, 20× magnification and laser lighting, we measure velocities from $10 \mu \mathrm{m} / \mathrm{s}$ up to $1 \mathrm{~m} / \mathrm{s}$. A typical velocity profile $v(z)$ for a half channel is shown in figure $2 \mathrm{~b}$ and could be used to probe the local rheology of the fluid $[4,5,8,31-33]$. We observe that the velocity does not vanish at the wall but tends to a constant $V$, which is precisely the slip velocity. We determine it by linear extrapolation of $v(z)$. The error on $V(\sim 10 \%-20 \%)$ is due to the measurement dispersion and the error on the wall position, taken equal to the diameter of the fluorescent markers $1 \mu \mathrm{m}$.

\section{RESULTS AND DISCUSSION}

Figure 3a shows measurements of the wall slip friction, that is, the slip velocity $V$ as a function of the wall stress $\sigma_{w}=\Delta P h /(2 L)$, for Carbopol 980 at different concentrations $c$. We observe that the slip velocity increases with the wall stress, as intuitively expected, and decreases with the polymer concentration. Besides, in these log log plots, we find that $V\left(\sigma_{w}\right)$ behaves as two straight lines of slopes $\approx 2$ and 1 at respectively low and large velocities. As expected from Eq.1, we observe a transition from an elastohydrodynamic to a Stokes-like regime. This transition is also seen for other types of Carbopol, as shown in Fig. 3b. Last, we find that the transition stress $\sigma^{*}$ at which the two lines meet increases with the concentration, which is also consistent with Eq.1.

Note that all measurements are performed for wall stresses above the yield stresses $\sigma_{Y}$, while previous measurements by Seth et al. [17] suggested a transition in slip regimes at $\sigma_{w} \approx$ $\sigma_{Y}$. In this stress range, we also detect no measurable wall yield stress, contrary to measurements from the recent literature $[10,17,21]$.

To go one step further, we now compare quantitatively our data to the predictions of Meeker et al. [18] (elastohydrodynamic friction $\left.\sigma_{\mathrm{EHD}}\right)$ and to Stokes friction $\sigma_{S t}$. Following [16], we assume that the total friction is the sum of both contributions $\sigma_{w}=\sigma_{\mathrm{EHD}}+\sigma_{\mathrm{St}}$ which can be recast as:

$$
\sigma_{w}=\sqrt{\sigma_{E} \frac{\eta V}{R}}+\alpha \frac{\eta V}{R}
$$




\begin{tabular}{|c|c|c|c|c|c|c|c|c|c|c|}
\hline Type & stirring & glycerol & $c$ & $\sigma_{Y}(\mathrm{~Pa})$ & $K\left(\mathrm{~Pa} \mathrm{~s}^{n}\right)$ & $n$ & $G^{\prime}(\mathrm{Pa})$ & $R(\mu \mathrm{m})$ & $c^{*}(\%)$ & $\eta(\mathrm{mPa} \mathrm{s})$ \\
\hline \multirow{5}{*}{ ETD } & & $0 \%$ & $1 \%$ & 15 & 5.0 & 0.58 & 60 & $0.72 \pm 0.15$ & \multirow{4}{*}{$0.07 \pm 0.01$} & \multirow{13}{*}{1} \\
\hline & strong & $0 \%$ & $0.5 \%$ & 4.1 & 4.4 & 0.52 & 26 & $0.84 \pm 0.08$ & & \\
\hline & & $0 \%$ & $0.25 \%$ & 2.0 & 2.5 & 0.51 & 15 & $1.01 \pm 0.17$ & & \\
\hline & & $0 \%$ & $0.1 \%$ & 0.8 & 1.6 & 0.51 & 7 & $1.66 \pm 0.20$ & & \\
\hline & weak (WS) & $0 \%$ & $0.5 \%$ & 12.5 & 7.3 & 0.49 & 65 & $1.82 \pm 0.36$ & $0.06 \pm 0.01$ & \\
\hline U10 & strong & $0 \%$ & $0.1 \%$ & 0.9 & 1.3 & 0.50 & 15 & $1.45 \pm 0.2$ & $0.09 \pm 0.01$ & \\
\hline \multirow{9}{*}{980} & & $0 \%$ & $0.3 \%$ & 62 & 20 & 0.37 & 370 & $0.85 \pm 0.08$ & \multirow{9}{*}{$0.08 \pm 0.02$} & \\
\hline & & $0 \%$ & $0.25 \%$ & 46 & 17.6 & 0.37 & 322 & $0.75 \pm 0.2$ & & \\
\hline & & $0 \%$ & $0.2 \%$ & 26 & 11 & 0.38 & 150 & $0.64 \pm 0.1$ & & \\
\hline & & $0 \%$ & $0.15 \%$ & 6 & 3.3 & 0.42 & 61 & $0.65 \pm 0.1$ & & \\
\hline & strong & $0 \%$ & $0.13 \%$ & 7.3 & 3.8 & 0.41 & 78 & $0.7 \pm 0.1$ & & \\
\hline & & $0 \%$ & $0.1 \%$ & 4.2 & 2.7 & 0.42 & 40 & $0.7 \pm 0.05$ & & \\
\hline & & $0 \%$ & $0.08 \%$ & 0.4 & 0.6 & 0.49 & 5.5 & $0.7 \pm 0.05$ & & \\
\hline & & $60 \%$ & $0.25 \%$ & 17 & 17.2 & 0.63 & 174 & $0.42 \pm 0.06$ & & \multirow[t]{2}{*}{10} \\
\hline & & $60 \%$ & $0.1 \%$ & 1.7 & 4.3 & 0.54 & 19 & $0.66 \pm 0.05$ & & \\
\hline
\end{tabular}

Table I. Bulk rheology parameters, particle radius $R$, jamming concentration $c^{*}$ and solvent viscosity $\eta$ for jammed suspensions prepared with different types of Carbopol, protocols (strong or weak stirring), glycerol concentrations and polymer weight concentrations $c$.
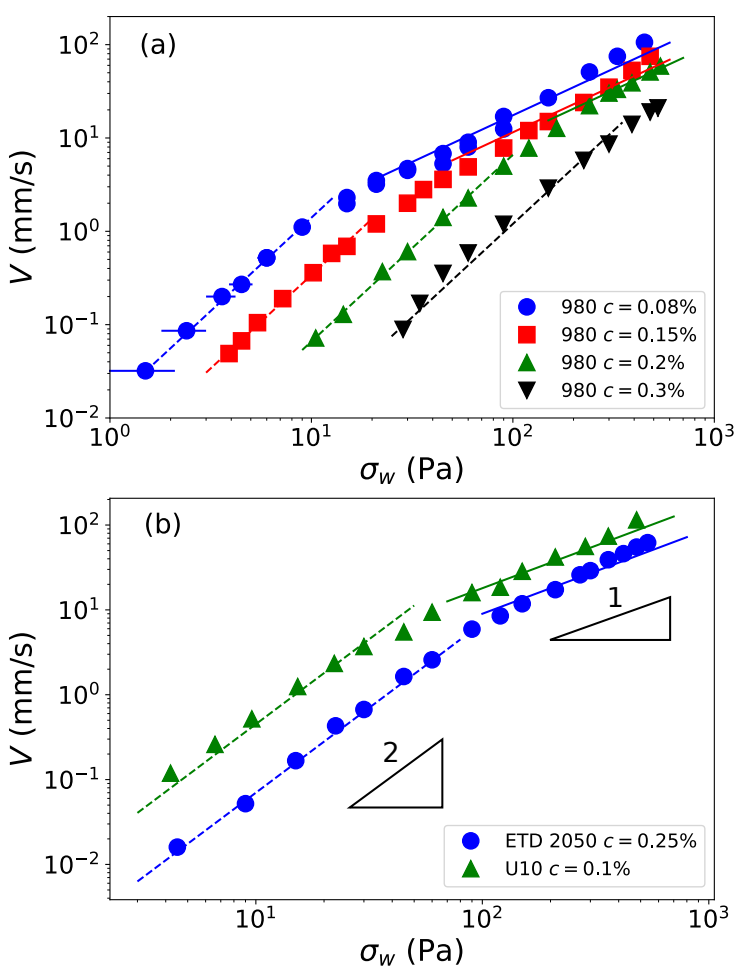

Figure 3. Wall friction: slip velocity $V$ vs the wall stress $\sigma_{w}$ (a) for Carbopol 980 at different concentrations and (b) for Carbopol ETD and U10 types. The straight lines correspond to $V \propto \sigma_{w}$ (solid lines) and $V \propto \sigma_{w}^{2}$ (dashed lines). Error bars are shown for Carbopol 980 at $c=0.08 \%$ and $c=0.3 \%$.

where $\sigma_{E}$ is an elastic stress characterizing the non-linear friction regime, while $\alpha$ is a dimensionless coefficient. To determine and compare
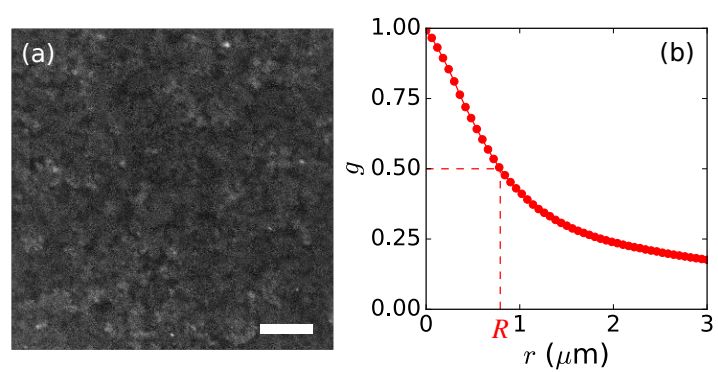

Figure 4. (a) Fluorescent image of Carbopol 980 at $c=0.15 \%$ obtained via confocal microscopy with Rhodamine 6G (scale $10 \mu \mathrm{m}$ ). (b) Normalized azimuthal average $g(r)$ of the spatial autocorrelation function of intensity fluctuations $\langle I(\mathbf{X}) I(\mathbf{X}+\mathbf{x})\rangle-\langle I(\mathbf{X})\rangle^{2}$, where $\langle\ldots\rangle$ denotes the average over $\mathbf{X} . g$ is normalized such that $\lim _{r \rightarrow 0} g(r)=1$.

both quantities, the radius $R$ of the polymer blob (particle) first needs to be determined.

Images of the microstructure are obtained via confocal microscopy. We incorporate in the microgel suspension Rhodamine $6 \mathrm{G}$ at final concentration $\sim 2 \mu \mathrm{M}$. The fluorescent cationic dye is attracted by the anionic polymer, which allows us to image the heteregenous structure of Carbopol at the micron scale (figure 4a). From the images, we calculate the normalized azimuthal average $g(r)$ of the spatial autocorrelation function of intensity fluctuations and determine the characteristic radius $R$ of the polymer particles, defined such that $g(r=R)=1 / 2$ (figure 4b). A similar procedure was used in [30]. Our measurements are summarized in table I. They show that $R$, in the range $0.4-2 \mu \mathrm{m}$, decreases with the concentration $c$ as the parti- 


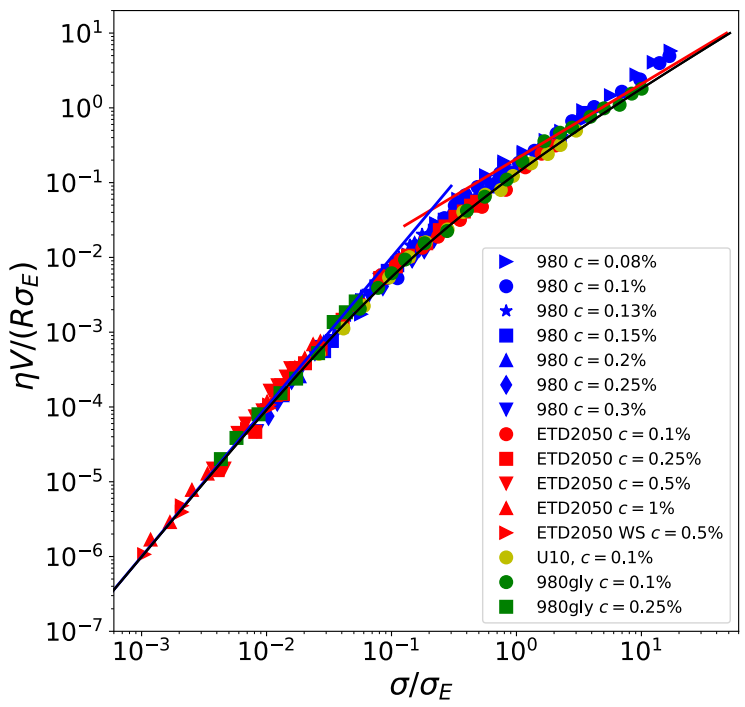

Figure 5. Dimensionless viscous stress $\eta V /\left(R \sigma_{E}\right)$ as a function of the normalized wall stress $\sigma_{w} / \sigma_{E}$ for the 15 Carbopol samples characterized in this study. $\sigma_{E}$ is extracted as a fit parameter from Eq.2. The black line corresponds to Eq.2 with $\alpha=\alpha_{\text {th }}=4.8$, the blue line to the elastohydrodynamic regime $\sigma^{2}=\sigma_{E}(\eta V / R)$ and the red line to the pure Stokes friction $\sigma=\alpha_{\mathrm{th}}(\eta V / R)$.

cles become more compressed and also that $R$ depends on the Carbopol type and the preparation protocol [30].

Once the radius of particles is known, we fit each data set (corresponding to a different sample) with Eq.2, and extract $\sigma_{E}$ and $\alpha$ as fit parameters. To show how our data compare to Eq.2, we use the fit parameter $\sigma_{E}$ to plot in figure 5 the dimensionless viscous stress $\eta V /\left(R \sigma_{E}\right)$ as a function of the dimensionless wall stress $\sigma_{w} / \sigma_{E}$. This representation allows us to collapse all our experimental results on a master curve, spanning 4 (resp. 7) orders of magnitude in dimensionless wall (resp. viscous) stress. Besides, we observe a very good agreement with the prediction of Eq.2, gathering data obtained with various Carbopol types, polymer concentrations $c$, preparation protocols and solvent viscosities $\eta$.

The master curve also highlights that the transition between both regimes is observed for $\sigma_{w}=\sigma^{*} \approx 0.2 \sigma_{E}$. Besides, we observe that the transition is very soft, spanning two orders of magnitude in both stresses and velocities. This explains why in other experiments, data in the intermediate regimes have been fitted as power laws with intermediate exponents [10]. This also highlights the importance of studying these friction regimes on many decades.

\section{A. Linear regime}

More quantitatively, we first estimate the expected friction coefficient $\alpha_{\text {th }}$ in the linear regime. If the force on each polymer sphere is Stokes-like $F=6 \pi \eta V R$, the average stress at the wall reads as $\sigma_{w}=\varphi F /\left(\pi R^{2}\right)=$ $(6 \varphi)(\eta V / R)$, with $\varphi$ the surface fraction of particles at the wall. This corresponds to $\alpha=6 \phi$. If we assume that particles are at the random close packing at the wall $(\varphi \approx 0.8)$, we find $\alpha_{\text {th }}=4.8$. This value is comparable to our fitted values of $\alpha$, whose average is 4.4 with a standard deviation of 1.2 for samples where the linear regime is present $\left(\sigma>0.2 \sigma_{E}\right)$. The theoretical value $\alpha_{\text {th }}$ has been used to plot the solid line shown in figure 5 which compares well to experimental data.

A more precise prediction of $\alpha$ is difficult due to the two following reasons. First, different cross-linking degrees depending on Carbopol type may result into a more or less porous microgel, hence different friction coefficients [34]. Second, the friction coefficient of the sphere should also depend on the distance of the sphere to the wall, as previously calculated by Chaoui and Feuillebois [35]. The fact that we find coefficients close to a simple Stokes friction, suggests that the particle-wall distance $\delta$ is then comparable to $R$, which is indeed confirmed by estimations from the elastohydrodynamic theory [18]: they predict $\delta / R \sim \sqrt{\eta V / R G_{p}}$. Approximating $G_{p}$ with the bulk elastic modulus of the suspension, we find $\delta / R \sim 0.3-1$ at the transition between the two slip regimes.

Nonetheless, it is striking that the simple Stokes argument allows us to predict the high stress wall friction of our Carbopol systems, whose microstructure is much more complex than monodisperse spheres. In particular, this highlights that the structural radius $R$ estimated from our microscopy measurements is comparable to the hydrodynamic radius in these jammed systems.

\section{B. Non-linear regime: elastic stress}

We now discuss the values of the elastic stress $\sigma_{E}$ characterizing the elasto-hydrodynamic regime at smaller velocities/stresses. In figure $6 \mathrm{a}$, we show $\sigma_{E}$ as a function of Carbopol mass concentration $c$ for the different samples used in this study. For each Carbopol type, we observe that the elastic stress $\sigma_{E}$ increases with $c$ as expected. We also report measurements from Meeker et al. [36] obtained for another type of microgel. They show a similar behav- 

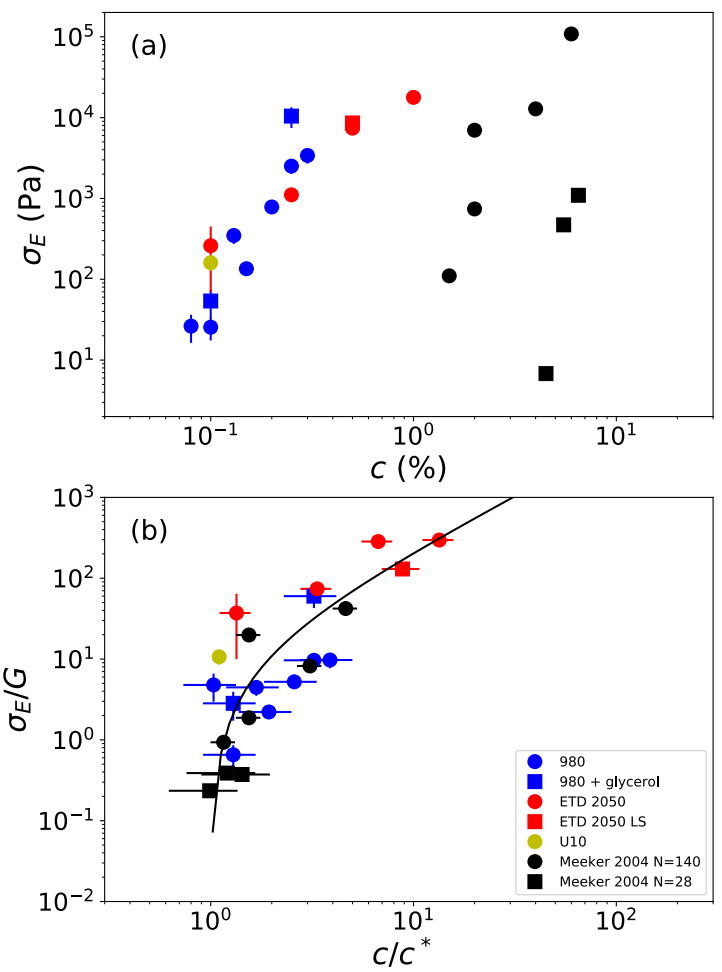

Figure 6. (a) Elastic stress $\sigma_{E}$ characterizing the elastohydrodynamic regime as a function of concentration $c$ for microgel samples exhibiting the non-linear elastohydrodynamic regime. $\sigma_{E}$ is extracted as a fit parameter from Eq.2. Data from [18] are also reported (black symbols). (b) Normalized elastic stress $\sigma_{E} / G$ as a function of the normalized concentration $c / c^{*}$. The solid line is a fit of Eq.3 $\left(\sigma_{E}=11\left(c / c^{*}-1\right)^{1.33}\right)$.

ior, yet at polymer concentrations $c$ larger by more than one order of magnitude.

More quantitatively, one expects from the model of Meeker et al. that $\sigma_{E} \sim$ $G_{P}\left(\Pi / G_{P}\right)^{4 / 3}$. This derivation is valid if the compressibility of the soft spheres is negligible, i.e. close to the jamming point, for $c^{*} \lesssim c$. In this limit, $G_{P}$ can be considered as roughly constant. Besides, the confinement pressure and the shear elastic modulus of a disordered packing of incompressible soft spheres as a function of the particle concentration have been numerically computed by Seth et al. [26]; their data can be empirically fitted as $\Pi / G_{P} \sim\left(c / c^{*}-1\right)^{k}$, with $k \approx 1.75$, and $G / G_{P} \sim\left(c / c^{*}-1\right)$. This yields

$$
\sigma_{E} \sim G\left(c / c^{*}-1\right)^{4 k / 3-1} \sim G\left(c / c^{*}-1\right)^{1.33} .
$$

To test our data against this prediction, we therefore plot in figure $6 \mathrm{~b} \sigma_{E} / G$ as a function of $c / c^{*}$. The jamming concentration $c^{*}$ is here determined by linear extrapolation of $c(G)$ data at low $G$. We find that this representation allows us to collapse our data with that obtained with other microgels [36] and are in reasonable agreement with Eq.3, even at concentrations well above jamming, up to $c \approx 10 c^{*}$, where the compression of microgels should not be negligible anymore.

Well above the jamming point $\left(c \gg c^{*}\right)$, we indeed expect the particles to be highly compressed: the film radius is then $r \sim R$, while the elastic pressure that sets the film thickness is simply the osmotic pressure $\Pi$, so that the characteristic elastic stress should be $\sigma_{E} \sim \Pi$. This osmotic pressure is predicted by the FloryRehner theory [27], yielding $\Pi \propto c^{9 / 4}$. In the same limit, the suspension shear modulus is given by that of the gel particles [27], hence proportional to the crosslink density $G \propto c$. This predicts $\sigma_{E} / G \propto c^{5 / 4}$. It should however be noted that the $5 / 4$ exponent is close to the one expected in the low concentration regime (1.33 for $c \gtrsim c^{*}$ ) so that both limiting behaviors may be difficult to delineate in practice. Being more quantitative would require independent characterizations of Carbopol spheres elastic modulus and osmotic pressure, beyond the scope of this study.

\section{CONCLUSION}

To conclude, we have demonstrated that the slip friction of jammed microgel suspensions exhibits a robust transition from a non-linear regime to a linear one at large wall stresses and slip velocities. We thereby reconcile seemingly contradictory measurements from the literature regarding slip laws of yield stress fluids above the yield stress $[5,8,17,21]$.

Combining microfluidic slip measurements to fluorescent imaging of the microgel structure, we are able to link both slip regimes to microscopic mechanisms. The linear friction is simply related to the Stokes flow past the particle, which dominates the dissipation at large velocities. We quantify the elastic stress which characterizes the non-linear elastohydrodynamic regime and relate it to the distance to jamming $c / c^{*}-1$.

While several studies have underlined that wall and bulk properties of yield stress fluids are intimately linked $[4,5,8,17,37]$, our results highlight that local arguments - at the particle scale - are sufficient to rationalize the friction law at the wall, where much insight can be gained on local dissipation mechanisms in yield stress fluids. Understanding the coupling between the dynamics of particles at the wall and in the bulk will prove necessary in the future to reconcile both points of view on soft 
glasses. At the microscopic level, our results also call for further studies of the poroelastic properties of individual microgel particles. Finally, existing models have never been tested at the microscale. This could be achieved by measuring the thickness of sheared films in the vicinity of the wall, which could be done with techniques using evanescent waves and total internal reflection [38-40].
Acknowledgements. We thank M. Cloitre for discussions and for sharing the data from [36]. We thank S. Manneville, E. Lorenceau, M. Leocmach, A.-L. Biance and T. Divoux for stimulating discussions, A. Piednoir for AFM measurements of wall roughness, V. Levy Dit Vehel for his help in the image analysis code and L. Jørgensen and Y. Pelet for rheological measurements used for the determination of $c^{*}$. We thank Institut Universitaire de France (IUF) for funding.
[1] R. G. Larson, The structure and rheology of complex fluids (Oxford University Press, New York, 1999).

[2] P. Coussot, "Yield stress fluid flows: A review of experimental data," Journal of NonNewtonian Fluid Mechanics 211, 31 (2014).

[3] D. Bonn, M. M. Denn, L. Berthier, T. Divoux, and S. Manneville, "Yield stress materials in soft condensed matter," Rev. Mod. Phys. 89, 035005 (2017).

[4] J. Goyon, A. Colin, G. Ovarlez, A. Ajdari, and L. Bocquet, "Spatial cooperativity in soft glassy flows," Nature 454, 84 (2008).

[5] B. Geraud, L. Bocquet, and C. Barentin, "Confined flows of a polymer microgel," European Physical Journal E 36, 30 (2013).

[6] H. A. Barnes, "A review of the slip (wall depletion) of polymer solutions, emulsions and particle suspensions in viscometers: its cause, character, and cure," J. Non-Newtonian Fluid Mech. 56, 221 (1995).

[7] M. Cloitre and R. T. Bonnecaze, "A review on wall slip in high solid dispersions," Rheol Acta 56, 283 (2017).

[8] V. Mansard, L. Bocquet, and A. Colin, "Boundary conditions for soft glassy flows: slippage and surface fluidization," Soft Matter 10, 6984 (2014).

[9] J.-B. Salmon, L. Bécu, S. Manneville, and A. Colin, "Towards local rheology of emulsions under Couette flow using Dynamic Light Scattering," Eur. Phys. J. E 10, 209 (2003).

[10] T. Divoux, V. Lapeyre, V. Ravaine, and S. Manneville, "Wall slip across the jamming transition of soft thermoresponsive particles," Physical Review E, Rapid Com. 92, 060301(R) (2015).

[11] Due to a weak attraction between the walls and the soft objects or a small roughness of the walls.

[12] X. Zhang, E. Lorenceau, P. Basset, T. Bourouina, F. Rouyer, J. Goyon, and P. Coussot, "Wall slip of soft-jammed systems: A generic simple shear process," Phys. Rev. Lett. (2017).

[13] N. D. Denkov, V. Subramanian, D. Gurovich, and A. Lips, "Wall slip and viscous dissipation in sheared foams: Effect of surface mobility," Colloids and Surfaces A 263, 129 (2005).

[14] S. Marze, D. Langevin, and A. Saint-Jalmes, "Aqueous foam slip and shear regimes determined by rheometry and multiple light scattering," Journal of Rheology 52, 1091 (2008).

[15] I. Cantat, "Liquid meniscus friction on a wet plate: Bubbles, lamellae and foams," Phys. Fluids 25, 031303 (2013).

[16] M. Le Merrer, R. Lespiat, R. Höhler, and S. Cohen-Addad, "Linear and non-linear wall friction of wet foams," Soft Matter 11, 368 (2015).

[17] J. R. Seth, C. Locatelli-Champagne, F. Monti, R. T. Bonnecaze, and M. Cloitre, "How do soft particle glasses yield and flow near solid surfaces?" Soft Matter 8, 140 (2012).

[18] S. P. Meeker, R. T. Bonnecaze, and M. Cloitre, "Slip and Flow in Soft Particle Pastes," Phys. Rev. Lett. 92, 198302 (2004).

[19] A. Poumaere, M. Moyers-González, C. Castelain, and T. Burghelea, "Unsteady laminar flows of a Carbopol@ gel in the presence of wall slip," Journal of Non-Newtonian Fluid Mechanics 205, 28 (2014).

[20] J. F. Ortega-Avila, J. Pérez-González, B. M. Marín-Santibáñez, F. Rodríguez-González, S. Aktas, M. Malik, and D. M. Kalyon, "Axial annular flow of a viscoplastic microgel with wall slip," Journal of Rheology 60, 503 (2016).

[21] X. Zhang, E. Lorenceau, T. Bourouina, P. Basset, T. Oerther, M. Ferrari, F. Rouyer, J. Goyon, and P. Coussot, "Wall slip mechanisms in direct and inverse emulsions," Journal of Rheology 62, 1495 (2018).

[22] H. M. Princen, "Osmotic Pressure of Foams and Highly Concentrated Emulsions. 1. Theoretical Considerations," Langmuir 2, 519 (1986).

[23] H. M. Princen and A. D. Kiss, "Osmotic Pressure of Foams and Highly Concentrated Emulsions. 2. Determination from the Variation in Volume Fraction with Height in an Equilibrated Column," Langmuir 3, 36 (1987).

[24] T. G. Mason, M.-D. Lacasse, G. S. Grest, D. Levine, J. Bibette, and D. A. Weitz, "Osmotic pressure and viscoelastic shear moduli 
of concentrated emulsions," Physical Review E 56, 3150 (1997).

[25] R. Höhler, Y. Yip Cheung Sang, E. Lorenceau, and S. Cohen-Addad, "Osmotic Pressure and Structures of Monodisperse Ordered Foam," Langmuir 24, 418 (2008).

[26] J. R. Seth, M. Cloitre, and R. T. Bonnecaze, "Elastic properties of soft particle pastes," Journal of Rheology 50, 353 (2006).

[27] P. Menut, S. Seiffert, J. Sprakel, and D. A. Weitz, "Does size matter? Elasticity of compressed suspensions of colloidal- and granularscale microgels," Soft Matter 8, 156 (2012).

[28] J. R. Seth, M. Cloitre, and R. T. Bonnecaze, "Influence of short-range forces on wall-slip in microgel pastes," Journal of Rheology 52, 1241 (2008).

[29] A. Huerre, O. Theodoly, A. M. Leshansky, M.P. Valignat, I. Cantat, and M.-C. Jullien, "Droplets in Microchannels: Dynamical Properties of the Lubrication Film," Phys. Rev. Lett. 115, 064501 (2015).

[30] B. Géraud, L. Jørgensen, C. Ybert, H. Delanoë-Ayari, and C. Barentin, "Structural and cooperative length scales in polymer microgels," Eur. Phys. J. E 40, 5 (2017).

[31] K. N. Nordstrom, E. Verneuil, P. E. Arratia, A. Basu, Z. Zhang, A. G. Yodh, J. P. Gollub, and D. J. Durian, "Microfluidic Rheology of Soft Colloids above and below Jamming," Phys. Rev. Lett. 105, 175701 (2010).

[32] K. N. Nordstrom, J. P. Gollub, and D. J. Durian, "Dynamical heterogeneity in softparticle suspensions under shear," Phys. Rev. E 84, 021403 (2011).
[33] P. Jop, V. Mansard, P. Chaudhuri, L. Bocquet, and A. Colin, "Microscale Rheology of a Soft Glassy Material Close to Yielding," Phys. Rev. Lett. 108, 148301 (2012).

[34] D. D. Joseph and L. N. Tao, "The effect of permeability on the slow motion of a porous sphere in a viscous liquid," ZAMM - Journal of Applied Mathematics and Mechanics / Zeitschrift für Angewandte Mathematik und Mechanik 44, 361 (1964).

[35] M. Chaoui and F. Feuillebois, "Creeping Flow around a Sphere in a Shear Flow Close to a Wall," The Quarterly Journal of Mechanics and Applied Mathematics 56, 381 (2003).

[36] S. P. Meeker, R. T. Bonnecaze, and M. Cloitre, "Slip and flow in pastes of soft particles: Direct observation and rheology," Journal of Rheology 48, 1295 (2004).

[37] T. Gibaud, C. Barentin, and S. Manneville, "Influence of Boundary Conditions on Yielding in a Soft Glassy Material," Physical Review Letters 101, 258302 (2008).

[38] R. Pit, H. Hervet, and L. Léger, "Direct Experimental Evidence of Slip in Hexadecane: Solid Interfaces," Physical Review Letters 85, 980 (2000).

[39] Z. Li, L. D'eramo, C. Lee, F. Monti, M. Yonger, B. Chollet, B. Bresson, Y. Tran, and P. Tabeling, "Near-wall nanovelocimetry based on Total Internal Reflection Fluorescence with continuous tracking," Journal of Fluid Mechanics 766, 147 (2015), arXiv: 1405.1372.

[40] A. Giuliani, R. McKenzie, and B. Loppinet, "Near wall velocimetry on a rheometer," Journal of Rheology 63, 93 (2019). 